\title{
The star formation rate in the inner Milky Way Galaxy
}

\author{
Oscar Cavichia ${ }^{1}$, Mercedes Mollá ${ }^{2}$, Roberto D. D. Costa ${ }^{1}$, and \\ Walter J. Maciel ${ }^{1}$ \\ ${ }^{1}$ IAG, University of São Paulo, 05508-900, São Paulo-SP, Brazil. \\ ${ }^{2}$ CIEMAT, Universidad Complutense de Madrid, Spain. \\ email: cavichia@astro.iag.usp.br
}

\begin{abstract}
The present star formation rate (SFR) in the inner Galaxy is puzzling for the chemical evolution models (CEM). No static CEM is able to reproduce the peak of the SFR in the $4 \mathrm{kpc}$ ring. The main reason is probably a shortage of gas, which could be due to the dynamical effects produced by the galactic bar, not considered by these models. We developed a CEM that includes radial gas flows in order to mimic the effects of the galactic bar in the first $5 \mathrm{kpc}$ of the galactic disk. In this model, the star formation (SF) is a two-step process: first, the diffuse gas forms molecular clouds. Then, stars form from cloud-cloud collisions or by the interaction between massive stars and the molecular gas. The former is called spontaneous and the latter induced SF. The mass in the different phases of each region changes by the processes associated with the stellar formation and death by: the SF due to spontaneous fragmentation of gas in the halo; formation of gas clouds in the disk from the diffuse gas; induced SF in the disk due to the interaction between massive stars and gas clouds; and finally, the restitution of the diffuse gas associated to these process of cloud and star formation. In the halo, the star formation rate for the diffuse gas follows a Schmidt law with a power $\mathrm{n}=1.5$. In the disk, the stars form in two steps: first, molecular clouds are formed from the diffuse gas also following a Schmidt law with $\mathrm{n}=1.5$ and a proportionality factor. Including a specific pattern of radial gas flows, the CEM is able to reproduce with success the peak in the SFR at $4 \mathrm{kpc}$ (fig. 1).
\end{abstract}

Keywords. Galaxy: abundances - Galaxy: evolution - Galaxy: formation.

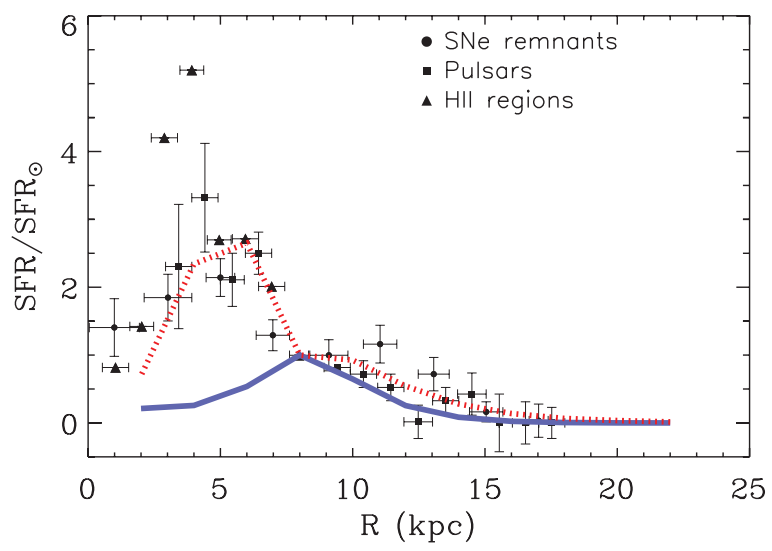

Figure 1. Present SFR as a function of the galactocentric distance. The observational data (circles, squares and triangles) are from Stahler \& Palla (2005, The Formation of Stars). The dashed and continuous lines represent the models with and without radial gas flows, respectively.

Acknowledgements: We acknowledge the support of FAPESP, CNPQ and CAPES. 